\title{
S/TEM Study of Fading Mechanism of Lithium Transition Metal Oxide Cathode for Lithium Ion Battery
}

\author{
Chongmin Wang, Pengfei Yan, Jianming Zheng, and Ji-Guang Zhang \\ Pacific Northwest National Laboratory, Richland, WA, USA
}

Although it has been observed that atomic layer deposition (ALD) coating of cathode particle with $\mathrm{Al}_{2} \mathrm{O}_{3}$ can significantly improve the battery performance, the exact functioning mechanism of such a thin layer of coating, in terms of surface chemistry between the cathode and electrolyte, is not sufficiently understood. The advanced scanning TEM (STEM) and spectroscopy techniques, X-ray energy dispersive spectroscopy (EDS) and electron energy loss spectroscopy (EELS) have been used to probe the functioning mechanism of $\mathrm{ALD}-\mathrm{Al}_{2} \mathrm{O}_{3}$ coating layer on lithium and manganese rich (LMR) cathode $\left(\mathrm{Li}_{1.2} \mathrm{Ni}_{0.2} \mathrm{Mn}_{0.6} \mathrm{O}_{2}\right)$. This work reports the direct visualization on the $\mathrm{Al}_{2} \mathrm{O}_{3}$ coating layer suppressing the cathode/electrolyte side reactions. The battery cycling induced severe $\mathrm{Mn}$ reduction that frequently occurred on uncoated cathode particle surface was significantly suppressed following the $\mathrm{Al}_{2} \mathrm{O}_{3}$ coating, therefore resulting in well-preserved particle surface and stabilized surface structure [1].

Battery cycling induced surface Mn reduction was perceived as one of the most important causes of cathode material degradation. Previous reports consistently showed that surface corrosion and surface layer structure transformation were closely related to Mn cation valence reduction in layered Mn-based cathode materials, because reduced $\mathrm{Mn}$ cations ( $\mathrm{such}$ as $\mathrm{Mn}^{2+}$ ) are believed to have higher mobility and can be easily dissolved into electrolyte. Moreover, the well-known Jahn-Teller effect will occur and destabilize the structure when Mn average valence state is reduced to 3+. Therefore, the stability of LMR cathode is heavily depended on Mn valence state. Thus, the Mn valence state and its spatial distribution on both uncoated and coated particle upon battery cycling were probed using STEM-EELS. Previous studies have established two methods can be used to estimate Mn valence state using EELS. One is through measuring Mn-L edge onset energy shift, so called chemical shift. The other is by calculating $\mathrm{Mn} \mathrm{L}_{3} / \mathrm{L}_{2}$ ratio. In this work, chemical shift was used to estimate Mn valence state, because it is more reliable due to its relative insensitive to sample thickness as well as the reliable processing protocol. In this case, Mn chemical shift was estimated by measuring $\mathrm{L}_{3}$ edge peak positions, because bulk EELS peak onset can be modified by the top and bottom surface layers, misleading the chemical shift measurement.

Figure 1 (b) and (e) show the measured Mn valence states for the uncoated and coated particles after 40 cycles, respectively. Distinctive difference can be seen on the spatial distribution of Mn valence between uncoated and coated particles. For the uncoated particle, the $\mathrm{Mn}^{2+}$ is uniquely located at the outmost surface of particle followed by a thick $\mathrm{Mn}^{3+}$ layer. In contrast, for the $\mathrm{Al}_{2} \mathrm{O}_{3}$ coated particle, $\mathrm{Mn}$ reduction only occurred in a very thin surface layer and their valence states are well above $3+$. Further, the EELS spectra acquired from surface and inner part of both uncoated and coated samples also support the mapping results (compare Figure 1(c) and (f)), where the chemical shift and $\mathrm{Mn} \mathrm{L}_{3} / \mathrm{L}_{2}$ ratio are distinctively different for the coated and uncoated samples. The spatial mapping of $\mathrm{Mn}$ valence distribution and its apparent correlation with the $\mathrm{Al}_{2} \mathrm{O}_{3}$ coating layer clearly indicates the $\mathrm{Al}_{2} \mathrm{O}_{3}$ coating suppresses the reduction of $\mathrm{Mn}$ at the cathode/electrolyte interface, therefore, mitigating the dissolution of the Mn into the electrolyte [2]. 
References:

[1] Pengfei Yan, et al, Chem. Mater 27 (2015), p. 7447.

[2] The work was conducted in the William R. Wiley Environmental Molecular Sciences Laboratory (EMSL), a national scientific user facility sponsored by DOE's Office of Biological and Environmental Research and located at PNNL.

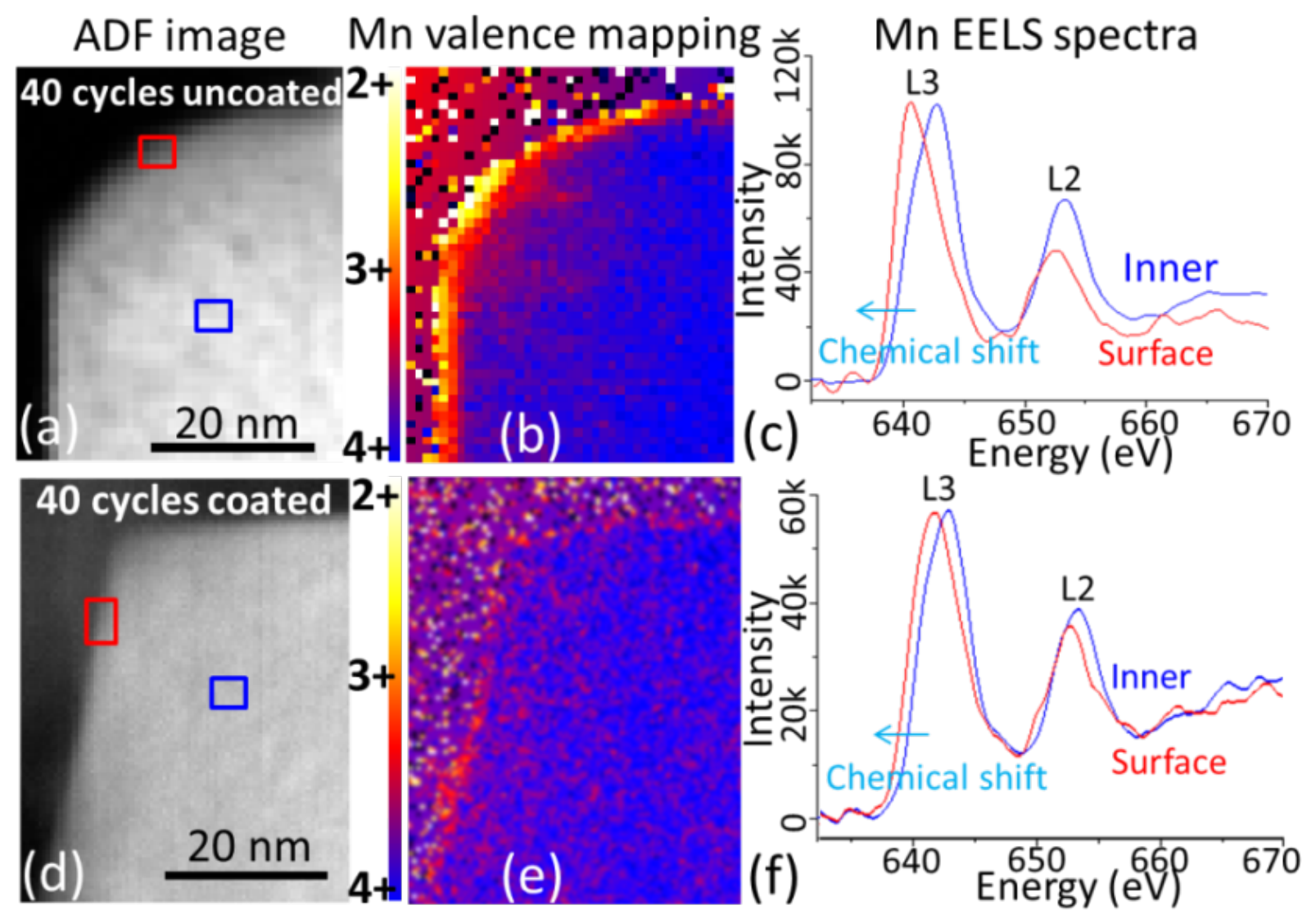

Figure 1. STEM-EELS analysis valence mapping of the 40 cycles and its correlation with the A12O3 coating layer. (a-c) uncoated and (d-f) coated samples. (a) ADF image and (b) estimated Mn valence state map based on EELS chemical shift. (c) Mn EELS spectra from surface and inner region in (a). (d) ADF image and (e) estimated Mn valence state map from EELS chemical shift. (f) Mn EELS spectra from surface and inner region in (d). 